\title{
Psicología cognitiva y neurociencias de la educación en el aprendizaje del lenguaje escrito y de las matemáticas
}

Cognitive psychology and neuroscience of education in learning the written language and mathematics

\author{
Luis Bravo Valdivieso ${ }^{1}$ \\ Pontificia Universidad Católica de Chile \\ (RECiBIDO 03/08/2014, AcEPTADo 10/10/2014)
}

\begin{abstract}
RESUMEN
Este artículo tiene por objetivo efectuar una revisión de las investigaciones actuales en psicología cognitiva y en neurociencias sobre el proceso de aprender el lenguaje escrito y las matemáticas.
\end{abstract}

Palabras clave: Neurociencias, psicología cognitiva, lenguaje escrito, cálculo, educación.

\section{SUMMARY}

The aim of this article is to describe the last researches in cognitive psychology and neurosciences for the learning of reading and mathematics.

Keywords: Neurociences, cognitive psychology, written language, calcule, education.

1 Profesor Emérito Universidad Católica de Chile Profesor Honorario. Universidad Nacional Mayor de San Marcos. Lima. E-mail: abravov @uc.cl 


\section{INTRODUCCIÓN}

El objetivo de este artículo es relacionar los aportes más actuales de la psicología cognitiva y de las Neurociencias con los procesos psicopedagógicos subyacentes al aprendizaje escolar del lenguaje escrito y de las matemáticas.

Una crítica que se hace con frecuencia a la Educación primaria es el alejamiento del progreso de las prácticas pedagógicas, del avance de las ciencias. Muchos maestros conservan celosamente "su estilo de enseñar" que generalmente es el mismo con el cual fueron ellos enseñados, pero no investigan y leen pocas investigaciones actualizadas sobre los procesos cognitivos y psicolingüísticos de cómo aprenden de los niños.

Las investigaciones en neuropsicología, datan desde la época de Luria (1966). Ellas muestran que no hay aprendizaje que no suceda en el cerebro y tampoco sin la mediación del lenguaje. Los resultados de algunas investigaciones más recientes confirman que el desarrollo cerebral infantil, presenta desde antes del nacimiento características que son determinantes de su desarrollo cognitivo y aprendizaje escolar. Con posterioridad al nacimiento se produce una integración de la influencia cultural y afectiva que aportan las escuelas y las familias con las bases biológicas y genéticas del cerebro. (Sigman, Peña, Goldin y Ribeiro ,2014 ; Guttorm, T.K., Leppanen, P.H.T., Richardson, U. y Lyytinen, H. 2001; Guttorm, T.K., Leppänen, P.H.T., Hämäläinen, J.A., Eklund, K.M. y Lyytinen, H.J. 2010; Molfese, D.L.2010). Esta integración les permite construir las estrategias cognitivas necesarias para aprender correctamente las enseñanzas escolares (para una revisiòn: Bravo 2014). El cerebro de los niños que ingresan al sistema pre escolar y escolar no es una "tábula rasa", pues trae características biológicas que son determinantes de su desarrollo. Estas variables que configuran el cerebro del niño, le permiten irse adaptando a la realidad exterior en la medida en que suceden los aprendizajes, en un proceso que Dehaene (2007) denominó "reciclaje neuronal". Las neuronas que reciben la información visual y auditiva del medio ambiente modifican sus conexiones sinápticas para procesar y asimilar los contenidos escolares, lo que ha sido especialmente investigado en el aprendizaje de la lectura y de las matemáticas.

Durante varios años, como consecuencia de los descubrimientos de Pavlov sobre los reflejos condicionados, de Skinner sobre el "condicionamiento operante" o de Piaget sobre el desarrollo cognitivo, muchas estrategias pedagógicas se centraron en el binomio "enseñanza- aprendizaje”. El proceso pedagógico podía resumirse en estrategias para "condicionar", "enseñar y evaluar" y "memorizar" contenidos o destrezas. La evaluación del aprendizaje exigía que los niños respondieran y memorizaran. Demoró bastante tiempo antes de que las escuelas de Pedagogía, que forman a los maestros, asumieran que los procesos cognitivos y psicolingüísticos de los niños también dependen del desarrollo y evolución biológica del cerebro. Sin embargo, hasta ahora no ha habido una integración satisfactoria entre las neurociencias y la psicología cognitiva con las estrategias de enseñanza psicopedagógica en los programas escolares. 
En un artículo sobre el aporte de las neurociencias a la educación, Sigman, Peña, Goldin y Ribeiro (2014) concluyeron que "los conceptos fundamentales del cerebro deberían ser parte del programa profesional de los maestros, de manera de proveerlos de un instrumento científico..." Expresan que el trabajo en "la construcción de relaciones directas entre la información del cerebro y las intervenciones pedagógicas prometen ser un campo importante de investigación para la futura neurociencias, como lo anticipó Hebb hace más de 60 años". De todas ha surgido el concepto de "neurociencias de la educación".

\section{NEUROCIENCIAS EDUCACIONAL}

Referente a las primeras investigaciones neuropsicológicas que mostraron la relación entre el cerebro y el lenguaje escrito es posible mencionar dos investigaciones seminales. En primer lugar, un descubrimiento clínico de Dejèrine (1887) en Francia, sobre las áreas cerebrales involucradas en lectura y escritura, conocimiento que ayudó a explicar muchas dificultades que tienen algunas personas para leer y escribir (Dehene,2007). La segunda, un siglo más tarde, fue de Galaburda y Kemper (1979) que descubrieron mediante anatomía patológica, que hay diferencias anatómicas en algunas áreas cerebrales entre sujetos que aprendieron a leer y otros que presentaron dificultades disléxicas. Estas investigaciones fueron continuadas por otras que confirmaron la relación entre las dificultades del aprendizaje y el desarrollo cerebral (Galaburda A., 1994).

Actualmente, las investigaciones más recientes son efectuadas con las nuevas técnicas de estudio del cerebro, (Resonancia Magnética Funcional IRMf, Potenciales Evocados P.E., Magneto encefalografía, Tomografía de emisión de positrones TEP y otras) las que confirman que el desarrollo cerebral infantil, tiene dos pilares fundamentales: el genético y las experiencias tempranas, especialmente relacionadas con el lenguaje, la percepción y el apego emocional. Sus descubrimientos han abierto nuevos caminos que obligan a reelaborar las estrategias educacionales, especialmente en la enseñanza inicial de las matemáticas y del lenguaje escrito.

Stern E., Grabner R., Schumacher R., Neuper Sc. y Saalbach H. (2005) efectuaron un extenso estudio sobre neurociencias pedagógicas y confirmaron que todos "los procesos cognitivos siempre se realizan en el cerebro". Agregan que "es indispensable un diálogo entre las neurociencias y la investigación educacional". Las investigaciones efectuadas hasta ahora muestran que el aprendizaje escolar, produce un aumento de la eficiencia neural, debido a que activa selectivamente algunas regiones del cerebro, lo que permite asimilar con mayor eficiencia diferentes informaciones y conocimientos. En consecuencia, la pedagogía de la lectura, de la escritura o de las matemáticas, debería tomar en consideración la estimulación de las áreas cerebrales involucradas, especialmente en el lenguaje, en la percepción visual y fonológica, en la orientación espacial y secuencial, en la memoria verbal y en la coordinación grafomotriz, para lograr aprendizajes más efectivos. 
Como consecuencia de estos descubrimientos, Szúcs D. y Goswami U. en 2007 propusieron el nombre de "Neurociencia Educacional" a un nuevo enfoque pedagógico que tiene por objetivo estudiar las "representaciones mentales en términos de la actividad neural del cerebro" (pág.114). Ella sería resultante de la combinación de "la neurociencia cognitiva y los métodos psicológicos para investigar el desarrollo de las representaciones mentales". (op.cit.,) y los métodos pedagógicos. La actual psicología cognitiva aparece como una ciencia intermediaria entre la biología y la educación. Las representaciones mentales implican "la actividad de la red neuronal del cerebro que codifica la información bajo la forma de actividad electroquímica". La neurociencia educacional tendría como objetivo descubrir como el cerebro elabora los procesos cognitivos, mediante "la interacción compleja de varias redes neuronales interconectadas" (op. cit).

Consideran que "la psicología cognitiva es el intermediario necesario entre la neurociencia y la educación" porque permite establecer estrategias psicopedagógicas de enseñanza basadas en cómo aprenden los niños. Expresan que "la psicología cognitiva ha mostrado las características esenciales de ciertas clases de representaciones mentales, cruciales para la educación, tales como las representaciones fonológicas para el aprendizaje alfabético y las representaciones de analogías de magnitud para el aprendizaje de los números". Del mismo modo como la psicología cognitiva ha descubierto que la clave del aprendizaje de la lectura está en la conciencia fonológica, la neurociencia cognitiva ha identificado un componente clave para el aprendizaje de las matemáticas, que es la representación mental de la magnitud (op.cit).

También Fawcett y Nicolson (2007) expresaron que las neurociencias pedagógicas, combinan los aportes de la psicología, la medicina, y la educación. Ellas integran los conocimientos sobre el cerebro con los procesos cognitivos y verbales, y su aplicación permite el estudio, diagnóstico y tratamiento de las dificultades de aprendizaje escolar. Por su parte, McCandliss B. (2010) expresa que la neurociencia del desarrollo cognitivo ha mostrado "el papel que juegan las experiencias educacionales para configurar los circuitos funcionales que originan las destrezas cognitivas tales como la lectura o las matemáticas". Concuerda en que "tales estudios señalan la emergencia de la neurociencia educacional".

En síntesis, las investigaciones actuales de la relación entre el procesamiento cerebral y el aprendizaje escolar son un aporte determinante para la psicopedagogía, la que no depende solamente de la aplicación de atinadas metodologías pedagógicas tradicionales, para enseñar, sino también de los procesos cognitivos y cerebrales que utilizan los niños para aprender. Los maestros no solo deben preocuparse de cómo enseñar sino de cómo los alumnos aprenden.

En consecuencia, la interrogante central de las neurociencias de la educación es ¿qué ocurre en el cerebro cuando el niño aprende? y ¿cómo evoluciona el cerebro con su aprendizaje? Preguntas cuyas respuestas conducen a elaborar estrategias pedagógicas que ayuden a los niños a aprender mejor y resolver sus dificultades de aprendizaje. 


\section{LA EVOLUCIÓN DEL APRENDIZAJE ESCOLAR}

Hemos visto que hasta la década de 1960 las metodologías pedagógicas de enseñanza del lenguaje escrito se centraban en desarrollar la percepción y la memoria visual de las letras y sílabas. La memoria visual era clave para asociar los signos gráficos y su pronunciación. El aprendizaje de las matemáticas, por su parte, se basaba en la memorización de las cifras, sus secuencias y su cálculo. Estas estrategias psicopedagógicas estimulaban principalmente los procesos de memoria repetitiva. Muchos niños que "aprendían a leer" solamente decodificaban o recordaban los signos ortográficos sin penetrar en su contenido o captar en ellos una sintaxis que les permitiera elaborar redes semánticas de comprensión. Los que tenían dificultades con las matemáticas, aprendían de memoria las tablas de multiplicar, pero no sabían cuando aplicarlas a problemas simples o más complejos como una "regla de tres". En ambos casos las enseñanzas se limitaban al desarrollo de aprendizajes instrumentales que no contribuían a desarrollar el pensamiento crítico y creativo.

En 1971 las investigaciones de Liberman y Shankweiler (1971) y en 1972 de Vellutino F., Steger J. y Kandel G. (1972) descubrieron que el problema común de muchos niños que no aprendían a leer no era que tuvieran un déficit de memoria visual, que les impidiera distinguir u orientar las letras y palabras, sino una insuficiencia fonológica. Tenían dificultad para discriminar los fonemas de las palabras y asociarlos con los signos ortográficos correspondientes lo cual limitaba su aprendizaje lector. En 2009 Fletcher consideró que el descubrimiento de la estructura fonológica había sido la clave para la enseñanza y el aprendizaje del lenguaje escrito, pues fue el que permitió establecer una relación causal entre el lenguaje oral y el lenguaje escrito y para dar significado a las palabras en la lectura (Alegría, 2006; Bravo 2011; Jiménez 2012). Las investigaciones en neurociencias más recientes han confirmado que esta relación entre los procesos visuales y los fonológicos se establecen en algunas áreas precisas del cerebro (Dehaene, 2007; Shaywitz y Shaywitz 2008; Fletcher 2009; Wimmer, Schurz, Sturm , Richlan , Klackl , Kronbichler y Ladurne 2010). Esta asociación muestra que el lenguaje escrito es un proceso cognitivo verbal y visual que contribuye a formar las "redes semánticas" que insertan al lector en un significado y también en una cultura. La asociación entre estos procesos está interferida en los niños disléxicos. En una propuesta sobre un "modelo cognitivo de la lectura", hemos considerado que habría que distinguir entre los procesos cognitivos periféricos, los procesos intermediarios y los procesos centrales (Bravo 2011). Las investigaciones mencionadas han mostrado que sus dificultades para leer están en algunos procesos cognitivos intermediarios, que establecen la conexión entre la percepción de los signos ortográficos y la pronunciación y significado de las palabras. Esta asociación permite transformar la información alfabética percibida visualmente en la comprensión de las palabras escritas y acceder al significado de la oración.

Stanovich (1988) expresa que las deficiencias para aprender se producen en un sistema cognitivo autónomo, que opera la decodificación del lenguaje escrito con independencia de los procesos cognitivos superiores. Considera que el procesamiento fonológico o la memoria operacional verbal, serían sistemas modulares 
autónomos, cuyo déficit no perturba el rendimiento intelectual aunque pedagógicamente sean responsables de un menor rendimiento cognitivo, como consecuencia del atraso que tienen los niños para aprender a leer.

Tunmer y Rohl (1991), asocian este déficit con las etapas del desarrollo del pensamiento infantil. Consideran que el aprendizaje de la lectura requiere previamente haber desarrollado una etapa metalingüística, que permita al niño reconocer sus propios procesos cognitivos y verbales. Es una capacidad de descentración, en sentido piagetano, que permite al niño oscilar el enfoque de su atención, desde las características visuales de los signos gráficos al fonémico, y expresarlo en su lenguaje oral. Una característica, tanto de las operaciones metalingüísticas, como de la “descentración”, está la habilidad para controlar el curso del propio pensamiento.

De estos estudios se desprende, que si bien la conciencia fonológica y la memoria operacional verbal son específicas y necesarias para el aprendizaje del lenguaje escrito, su efectividad también afecta el desarrollo cognitivo global. En consecuencia, "Aprender a leer" no es solamente aprender a reconocer y decodificar la escritura, sino aprender a atribuir significado al texto alfabético y establecer una metacognición de los componentes cognitivos del lenguaje escrito. (Bravo, 2013). En consecuencia, junto con reconocer que la escritura tiene un significado es necesario que los niños elaboren estrategias para encontrar su significado y transformar así su pensamiento en cultura alfabética. Este proceso denominado metacognición les permite reconocer e intervenir sobre los propios procesos cognitivos con los que está aprendiendo. Por esta razón aparece más conveniente emplear el término "aprendizaje del lenguaje escrito" que simplemente aprender a leer. La lectura es una segunda clave del lenguaje como planteó Vitgotsky y el historiador James Dunn expresa que "el texto no hace el significado, los lectores hacen el significado" (2003 pag.95).

En este proceso cognitivo Landern, Frith y Wimmer (1996) observaron que en el aprendizaje normal del lenguaje escrito se produce una coactivación de las representaciones fonológicas y ortográficas. Esta coactivación es más débil en los niños disléxicos, los que tienen menor conexión entre ellas. Al ver una palabra escrita no logran evocar automáticamente su sonido. Tampoco la percepción de los fonemas les evoca la imagen ortográfica de las letras o sílabas. Su investigación planteó un interesante problema neuropsicológico que ha venido a ser resuelto después del año 2000: ¿En qué parte del cerebro de los niños disléxicos se produce la interferencia que debilita el reconocimiento y aprendizaje del lenguaje escrito y la asociación entre los procesos visuales ortográficos con los procesos auditivos fonológico? La respuesta hubo que buscarla en las neurociencias.

\section{EL APORTE DE LAS NEUROCIENCIAS}

Hemos visto que en el presente siglo XXI se ha producido una renovación del conocimiento sobre el aprendizaje del lenguaje escrito y de las matemáticas debido al aporte de las neurociencias (Bravo, 2014). 
Dehaene (2007) coincide en que los progresos de las neurociencias y de la psicología cognitiva han originado el nacimiento de una "verdadera neurociencia de la educación en la frontera entre la psicología y la medicina, capacitada para aprovechar las nuevas imágenes del cerebro con la finalidad de optimizar las estrategias de enseñanza y adaptarlas a cada cerebro de niño o de adulto" (pag. 22).

Una condición para el éxito en la aplicación pedagógica de este proceso es conocer cómo el cerebro activa selectivamente algunas regiones frente a los estímulos y determina sus conexiones. Dehaene (op.cit) considera que durante el aprendizaje se produce en el cerebro del niño un progresivo "reciclaje neuronal", que le permite, entre otras actividades, asimilar, retener y elaborar los nuevos conocimientos.

Numerosos investigadores, como Shaywitz S., Shaywitz B., Pugh K., Fulbright R., Constable, y Mencl W. al (1998); Simos, Fletcher, Safari, Billingsted Marshall (2007), Shaywitz y Shaywitz (2008), Fletcher J., Lyon G., Fuchs L. y Barnes M. (2007), Fletcher (2009), han mostrado que los nuevos aportes de las neurociencias permiten conocer mejor la compleja interacción entre el desarrollo cerebral con los estímulos lingüísticos, sociales y emocionales, originados por el ambiente escolar y familiar.

Un ejemplo de estas investigaciones donde se integran los aportes cognitivos y neurocientíficos, es la investigación de Stern E., Grabner R., Schumacher R., Neuper C. y Saalbach H. (2005) que han mostrado que los niños con déficit fonológico muestran una menor actividad en las áreas cerebrales témporo-parietales izquierdas cuando tienen que dar una respuesta acerca del ritmo de las letras y sílabas. Luego, al mejorar su aprendizaje de la lectura, el cerebro aumenta también su actividad en estas áreas.

Por su parte, Simos, Fletcher, Bergman, Breier, Foorman, Castillo y Davis (2002) mostraron que luego de un periodo de intervención psicopedagógica en niños con retardo para aprender a leer, junto con el aprendizaje aparecía una "normalización" en los circuitos cerebrales que procesan la lectura. Una consecuencia de esta investigación es que las dificultades en lectura pueden ser corregidas mediante un mejor desarrollo del procesamiento fonológico asociado con el reconocimiento visual de las letras. Sus investigaciones mediante imágenes cerebrales de resonancia magnética (IRMf) mostraron que también algunas deficiencias en las conexiones cerebrales pueden ser superadas con la intervención en los procesos de conciencia fonológica y en el reconocimiento visual ortográfico de las letras. Posteriormente, Simos, Fletcher, Safari, Billingsted-Marshall, Denton, y Papanicolau, (2007) confirmaron este resultado en otra intervención pedagógica a niños con dificultades severas de lectura, de edad entre 7 y 9 años. Concluyeron que en los niños en los que se efectuó la intervención psicológica mostraron clara mejoría, tanto en la conciencia fonológica como en el reconocimiento visual de las palabras y que la instrucción intensiva a nivel de las palabras efectuó cambios neurofisiológicos significativos (op.cit.). También Shaywitz (2003), mostró que la intervención en la activación de la conciencia fonológica, produjo modificaciones en las imágenes cerebrales de niños con retardo lector. Luego del tratamiento, sus imágenes cerebrales aparecían semejantes a los lectores normales. 
Stern, Grabner, Schumacher, Neuper y Saalbach (2005) concluyeron que las investigaciones neurocientíficas pueden tener importancia psicológica y pedagógica pues aportan evidencia que hay causas neuronales en algunos déficits del aprendizaje. También mostraron que los déficits cognitivos y del aprendizaje pueden tener distintos orígenes neuronales, los cuales no pueden ser observados directamente, en cambio los estudios neurofisiológicos, " pueden identificar diferentes causas de un déficit cognitivo particular en distintos individuos" (op.cit). Concluyeron que las investigaciones neurocientíficas tienen trascendencia psicológica y pedagógica, pues permiten descubrir los orígenes y las diferencias entre algunos transtornos del aprendizaje que no son observables en el nivel escolar.

Una consecuencia de la integración de las investigaciones en neurociencias con el proceso educativo lo expresó Fletcher (2009) diciendo que el proceso de "Aprender a leer, literalmente reescribe la organización del cerebro" por cuanto el sistema neural subyacente al reconocimiento de las palabras es maleable. En consecuencia, aunque las dificultades para aprender tengan un origen temprano eso no impide que algunos niños puedan establecer mecanismos compensatorios o alternativos, como consecuencias de una labor pedagógica intensiva. En estos casos no basta con aplicar metodologías de enseñanza corrientes, sino que es necesario integrarlas con estrategias destinadas a desarrollar la conciencia fonológica, semántica, sintáctica y alfabética en una metacognición del lenguaje escrito.

Respecto al tratamiento psicopedagógico de las dificultades lectoras, un número importante de investigaciones han observado que el progreso en el aprendizaje es mayor y más duradero si la intervención es temprana y sigue patrones derivados de una estrategia centrada en el desarrollo fonológico y visual-ortográfico (Torgesen J.,Alexander A., Wagner R , Rashotte C, Voeller K ,Conway T. 2001; Cuadros 2010).

Desde el punto de vista de los descubrimientos científicos de las neurociencias relacionados con la educación, Dehaene (2007, pag.30) expresa que "el ejemplo de la lectura muestra que la organización cultural está ligada inextricablemente a la de nuestro cerebro. En el curso de su larga historia cultural, la humanidad ha descubierto que ella podía reconvertir su sistema visual a fin de reconocer la escritura" y él describe admirablemente los procesos que efectúa el cerebro para lograr el aprendizaje del lenguaje escrito y de las matemáticas.

Mac Candliss (2010) también considera que las neurociencias educacionales requieren investigar los cambios que se producen en el cerebro con el aprendizaje. Un ejemplo ha sido el descubrimiento de la relación entre el proceso de asociar la percepción ortográfica de las palabras con sus correspondientes fonemas. Las investigaciones con FMRI han mostrado que para que se origine el aprendizaje lector es indispensable que la asociación entre las letras y los fonemas correspondientes ocurra en una determinada región cerebral. Agrega que los niños con dislexia, tienen dificultad para efectuar esta asociación entre los sistemas neuronales que conducen ambos procesos. La intervención educacional puede facilitar esta asociación y el reconocimiento de las palabras. También Wimmer, H., Schurz, M., Sturm, 
D., Richlan, F., Klackl, J., Kronbichler, M. et al. (2010), mediante la técnica de FMRI confirmaron que la deficiencia en la conexión visual ortográfica y fonológica observada en un grupo de disléxicos de habla alemana podría estar originadas en alteraciones en el desarrollo de algunos procesos neuropsicológicos subyacentes, fallas que pueden corregirse mediante la intervención psicopedagógica.

Como consecuencia de estas investigaciones Goswami U, (2004) concluyó que "es indispensable un diálogo entre las neurociencias y la investigación educacional” (pag. 32).

\section{EL APRENDIZAJE DE LAS MATEMÁTICAS Y LOS PROCESOS NEUROCOGNITIVOS}

Las investigaciones en Matematicas también han mostrado, en estudios mediante EEG, que su aprendizaje también produce modificaciones en el desarrollo cerebral lo que implica que la Educación debe considerar el procesamiento cerebral para la enseñanza del cálculo. Dehaene (2009) considera que la intuición de los números, sus adiciones y sustracciones que están presente en todas las culturas humanas está relacionado con el lóbulo parietal, en un área localizada en el surco intraparietal de ambos hemisferios. Agrega que un "próximo desafío será comprender como la educación puede modificar nuestras intuiciones básicas de los números".

Así como la neuropsicología permitió descubrir las claves cerebrales del aprendizaje del lenguaje escrito, también ha contribuido a explicar algunos procesos del aprendizaje de las matemáticas. Especialmente han sido importantes las investigaciones de Dehaene S., Piazza M, Pinel Ph, y Cohen L. (2003) y Piazza, M., Pinel, P., Le Bihan, D., et al. (2007) y Dehaene, S., y Cohen, L. (1995). Los primeros propusieron que desde el punto de vista cognitivo habría tres tipos de representaciones numéricas: las verbales, las semánticas no-verbales y las viso-espaciales. En su aspecto verbal, ellas también dependen del procesamiento fonológico - pues para nombrar las cifras hay que verbalizarlas - y algunas deficiencias para aprender pueden estar relacionadas con las mismas deficiencias fonológicas que se encuentran en las dislexias.

Agregan que el triple código del procesamiento numérico depende de las tareas, en las cuales se observan tres sistemas de representaciones diferentes: “ ...un sistema cuantitativo (no verbal semántico de representación del tamaño y distancia entre los números)... un sistema verbal (donde los numerales son representados lexicalmente, fonológicamente y sintácticamente, semejante a las demás palabras) y un sistema visual en el cual los números pueden ser codificados como series de numerales arábicos".

Dehaene y col. (2003) establecen que hay relación entre algunas áreas cerebrales del lenguaje y las que intervienen en el cálculo matemático. Desde el punto de vista cerebral considera que "la contribución del giro angular cerebral izquierdo en el procesamiento de los números puede relacionarse con la base lingüística del cálculo matemático" (op.cit). También consideran que una deficiente conectividad funcional en el "giro angular izquierdo" del cerebro es consistente con la dificultad para recordar los números lo cual muestra que el aprendizaje de los números tiene una base en la evolución neurológica del cerebro. 
Dehaene (2003) especificó que la región cerebral denominada "Surco horizontal intra parietal , HIPS" ( Horizontal Intra Parietal Sulcus) parece activarse cuando una operación aritmética requiere una representación numérica cuantitativa. Aparece más activa cuando los sujetos hacen cálculos, que cuando solamente tiene que leer números, lo cual indica que incide en el procesamiento semántico de los números".

Además sugieren que el HIPS (Horizontal segment of intraparietal sulcus) es esencial para la representación semántica de los números como cantidades. Esta representación puede proporcionar un fundamento para nuestra "intuición numérica", la cual a menudo es la comprensión inconsciente e inmediata de la cantidad. (Dehaene, 1992, 1997; Dehaene y Marques, 2002)"

Sin embargo, Dehaene y col. (2003) también expresan que "El giro angular izquierdo del cerebro no parece implicado con el procesamiento cuantitativo, pero muestra mayor activación cuando la tarea requiere del procesamiento verbal. Por consiguiente "Contribuye al procesamiento de los números cuando las operaciones aritméticas requieren de la codificación verbal de los números, como sucede en la multiplicación" Esta hipótesis se apoya en los descubrimientos que muestran que "el giro angular izquierdo está implicado en diferentes procesos verbales tales como la lectura o la memoria verbal de corto alcance". En síntesis , la contribución del giro angular izquierdo en el procesamiento de los números puede relacionarse con las bases verbales del cálculo aritmético, especialmente en la memoria verbal de los números.

Los resultados de sus investigaciones coinciden con la noción que en el cerebro se desarrolla un sistema de números asociados en la corteza intra parietal bilateral , que sería igual en sujetos de todas las culturas, además de un circuito peri silviano asociado con el lenguaje . También consideran que el lóbulo parietal contribuye a la representación de las cantidades en "una línea mental numérica". Consideran que la educación aporta algunas estrategias específicas para almacenar y recuperar la información aritmética (Dehaene y Cohen 1995).

Dehaene (2009) considera que la actividad numérica del área parietal parece estar funcionando antes de la enseñanza de la aritmética. "El procesamiento parietal funcional del sistema de los números aparecería a una edad muy temprana durante la infancia". " Los estudios mediante FMRI han demostrado que el surco intraparietal está más activado durante las tareas que requieren manipular cantidades particularmente aproximaciones de adiciones y sustracciones"

También expresa que tanto el lenguaje como la aritmética están lateralizados en el hemisferio izquierdo en la mayoría de las personas diestras. Su "modelo de desarrollo sugiere que esta "colateralización" refleja una interacción causal durante el desarrollo, la lateralización del lenguaje precede y origina una progresiva lateralización de las representaciones numéricas en el lóbulo parietal" (2009)

En conclusión, la cantidad de investigaciones actuales que nos aportan la Psicología Cognitiva y las Neurociencias destinadas a conocer el aprendizaje, abren a la Educación una nueva vía de conocimientos que le permiten profundizar las 
principales variables del desarrollo infantil desde el punto de vista de los procesos cerebrales donde se originan sus aprendizajes. Aparece evidente la necesidad de investigaciones conjuntas e integradas de estas tres ciencias conducentes plantear nuevos modelos teóricos educativos para la enseñanza inicial. Einstein expresó en una oportunidad que las teorías en las ciencias son una elaboración del espíritu. Por medio de ellas los investigadores pretenden explicar de manera coherente e integrada las hipótesis y los resultados que subyacen a cada investigación. Las Neurociencias, como hemos visto, han permitido en los últimos años elaborar nuevas teorías sobre los procesos subyacentes al aprendizaje escolar. Ellas permiten entrelazar la motivación de los investigadores con la de los educadores mediante la aplicación de renovadas metodologías pedagógicas y psicológicas y proponer interpretaciones de los resultados obtenidos. La Educación debería elaborar un modelo educacional que se centrara en determinar cómo los niños aprenden en sus cerebros y cuáles serían las estrategias psicopedagógicas y neuropsicológicas más adecuadas para este aprendizaje,

\section{REFERENCIAS BIBLIOGRÁFICAS}

Alegría J. (2006). Por un enfoque psicolingüístico del aprendizaje de la lectura y sus dificultades - 20 años después. Infancia y Aprendizaje 29: 93-111.

Bravo L. (2011). Lenguaje escrito y dislexias: Enfoque cognitivo del retardo lector. Quinta Edición. Santiago de Chile. Ediciones Universidad Católica.

Bravo L. (2014). Neurociencias y Educación : Estado actual de la investigación en Dislexias. Estudios de Psicología, 35, 1, 1-28.

Cuadros A. (2010). La lectura y sus dificultades: Dislexia Evolutiva. Grupo magro Editores. Montevideo.

Dehaene, S., y Cohen, L. (1995). Towards an anatomical and functional model of number processing. Mathematical Cognition., 1, 83-120.

Dehaene S. (2009) Origins of Mathematical Intuitions. The Case of Arithmetic. The Year in Cognitive Neuroscience 2009: Annual New York Academy of Science. 1156: 232259. doi: 10.1111/j.1749-6632.2009.04469.

Dehaene S. (2007). Les neurones de la lecture. Paris. Odile Jacob.

Dehaene S., Piazza M, Pinel Ph, y Cohen L. (2003). Three Parietal circuits for number processing. Cognitive Neuropsychology, 20, 487-506.

Dejèrine J. (1887).Contribution à l'étude anatomo-pathologique et clinique des diferentes variétés de cecité verbale. Mémoires de la Société de Biologie 4, 61-90.

Dunn J. (2003). El Cristianismo. Vol. 1. Wm. B. Eerdmans Publishing Co.

Fawcett A. y Nicolson R. (2007). Dislexia, aprendizaje y neurociencia pedagógica. Developmental Medecine and Child neurology, 49: 306-311.

Fletcher J. (2009). Dyslexia: The evolution of a scientific concept. Journal of the International Neuropsychological Society, 15, 501-508. 
Fletcher J., Lyon G., Fuchs L. y Barnes M (2007). Learning Disabilities: From Identification to intervention. New York. Guilford.

Galaburda A. y Kemper (1979). Cytoarchitectonic abnormalities in developmental dyslexia: A case study. Annals of neurology 6: 94-100.

Galaburda A. (1994). Developmental dyslexia and animal studies: at the interface between cognition and neurology. Cognition 50, 133-149.

Goswami U.( 2004). Neuroscience and Education. British Journal of Educational Psychology 74: 1-14.

Guttorm, T.K., Leppanen, P.H.T., Richardson, U. \& Lyytinen, H. Event-related potentials and consonant differentiation in newborns with familial risk for dyslexia. J. Learning. Disabilitiesl (2001) 34, 534-544.

Guttorm, T.K., Leppänen, P.H.T., Hämäläinen, J.A., Eklund, K.M. \& Lyytinen, H.J. Newborn event-related potentials predict poorer pre-reading skills in children at risk for dyslexia. J. Learning. Disabilities (2010) 43, 391-401.

Jiménez J.E.(Coord.) (2012). Dislexia en español. Madrid. Ed. Pirámide.

Landern,K. Frith U. y Wimmer H. (1996). Intrusion of orthographic knowledge on phoneme awareness: Strong in normal readers, weak in dyslexic readers Applied Psycholiguistics, 62.

Liberman I. y Shankweiler D. (1971). Letter confusions and reversals of sequence in the beginning reader. Cortex, 7: 127-142.

Luria A.R. (1966). Human brain and Psychological processes. New York. Harper and Row.

Mc Candliss, B.D. (2010). Educational neuroscience: The early years PNAS, vol. 107, $\mathrm{N}^{\mathrm{0}} 18,8049-8050$.

Molfese, D.L. Predicting dyslexia at 8 years of age using neonatal brain responses. Brain Langage. 72, 238-245 (2000).

Piazza, M., Pinel, P., Le Bihan, D., et al. (2007). A magnitude code common to numerosities and number symbols in human intraparietal cortex. Neuron, 53, 293-305.

Shaywitz S, Shaywitz B., Pugh K., Fulbright R., Constable y Mencl W. al. (1998). Functional disruption in the organization of the brain for reading in dyslexia.

Proceeding National Academy Science. USA, 95:2636-2641Shaywitz S. y Shaywitz B. (2008). Paying attention to reading: The neurobiology of reading and dyslexia. Developmental Psychopathology, 20,1329-1349.

Sigman M., Peña M., Goldin A. y Ribeiro S., (2014). Neuroscience and education: prime time to build the bridge. Nature neuroscience 17, 4: 497-502.

Simos P.G., Fletcher J.M., Bergman E., Breier J.,Foorman B.,Castillo E., Davis M. y col. (2002). Dislexia-specific brain activation profile becomes normal following successful remedial training. Neurology, 58: 1203-1213.

Simos P.G., Fletcher J., Safari , Billingsted- Marshall R., Denton C. y Papanicolau A. (2007). Intensive instruction affects brain magnetic activity associated with oral 
word reading in children with persistent reading disabilities. Journal of Learning disabilities 40:37-48.

Stanovich (1988). The right and wrong places to look for the cognitive locus of reading disability. Annals of Dyslexia 38:154-155.

Stern E., Grabner R., Schumacher R., Neuper C. y Saalbach H. (2005). Educational Research and Neurosciences-Expectations, Evidence, Research Prospects Education Reform. Vol. 13.

Szúcs D. y Goswami U. (2007). Educational Neuroscience: Defining a new discipline for the study of mental representations. Mind ,Brain and Education, 1:114-127.

Torgesen J., Alexander A., Wagner R , Rashotte C, Voeller K ,Conway T. (2001). Intensive remedial instruction for children with severe reading disabilities: Inmediate and long-term outcomes from two instructional approaches. Journal of Learning Disabilities, 34: 33-58.

Tunmer W.E y Rohl M. (1991). Phonological awareness and reading acquisition. En: D.J. Sawyer y B.J. Fox (Eds.) Phonological awareness in reading. Heildelberg. Springer Verlag.

Vellutino F., Steger J. y Kandel G. (1972). Reading disability: An investigation of the perceptual deficit hypotesis. Cortex 8: 106-118.

Wimmer, H., Schurz, M.,Sturm, D., Richlan, F., Klackl, J., Kronbichler, M. et al. (2010).A dual-route perspective on poor reading in a regular orthography: An fMRI study. Cortex, DOI: 10.1016/j.cortex.2010.06.004. 